\title{
NEAR-INFRARED OBSERVATIONS OF THE PROTO-PLANETARY NEBULA AFGL 618
}

\author{
WILLIAM B. LATTER ${ }^{1}$ \\ Canadian Institute for Theoretical Astrophysics, University of Toronto, McLennan Physical Labs, Toronto, Ontario, Canada M5S 1A1
}

Philip R. MALONEY

NASA/Ames Research Center, MS 245-6, Moffett Field, CA 94035, and Sterrewacht Leiden, 2300 RA Leiden, The Netherlands

AND

Douglas M. Kelly, John H. Black, G. H. Rieke, and M. J. Rieke

Steward Observatory, University of Arizona, Tucson, AZ 85721

Received 1991 March 4; accepted 1991 October 11

\begin{abstract}
We present near-infrared images and spectroscopy of the bipolar proto-planetary nebula AFGL 618. Emission from molecular hydrogen is detected in the $0.90-1.34 \mu \mathrm{m}$ spectrum. From molecular hydrogen line ratios, we derive a visual extinction of $A_{V}=3.4_{-2.0}^{+4.0} \mathrm{mag}$ to the $\mathrm{H}_{2}$-emitting region. Models of collisionally excited and fluorescent $\mathrm{H}_{2}$ emission are compared with the data: the near-infrared $\mathrm{H}_{2}$ spectrum is dominated by emission from collisionally excited molecules at $T_{\mathrm{ex}} \approx 2000 \mathrm{~K}$, but a component of fluorescent emission is also present. The low level of fluorescent emission might indicate a clumpy structure, or one which partly shields the emitting region from the stellar continuum. We show that this type of near-infrared spectrum is an excellent tool for discriminating relatively low levels of fluorescent $\mathrm{H}_{2}$ emission from a strong collisionally excited component.

Images in the $\mathrm{H}$ - and $\mathrm{K}$-bandpasses have been analyzed using models which assume single scattering of photons from the central object. The models indicate that the bipolar axis is inclined to the plane of the sky by $i \sim 45^{\circ}$. An unresolved, compact source is seen in both images and is reproduced in the models. It is found that the emission in the $\mathrm{H}$ - and $\mathrm{K}$-bands is consistent with single scattering of photons by dust with a distribution such that the dust density decreases with increasing stellar latitude and is nearly zero at the poles. A total visual attenuation at the stellar equator of $A_{V} \sim 87 \mathrm{mag}$ is required to reproduce the images.
\end{abstract}

Subject headings: infrared: interstellar: continuum - infrared: interstellar: lines - molecular processes planetary nebulae: individual (AFGL 618)

\section{INTRODUCTION}

As stars evolve off the asymptotic giant branch (AGB) on the HR diagram to become planetary nebulae, they sometimes (perhaps always) take on the observed form of bipolar nebulae (e.g., Zuckerman \& Gatley 1988). Such objects are characterized by symmetric lobes of gas and dust flowing outward from an obscured central star. This apparently brief period in a star's life (Morris 1981; Jura \& Kroto 1990) is also one of the least understood. It has been suggested that bipolar nebulae result from stellar mass-loss in a close asynchronous binary system (Livio, Salzman, \& Shaviv 1979; Morris 1981, 1987; Bond \& Livio 1990). While there is good reason to believe that such duplicity shapes at least some bipolar nebulae, it might not be the only mechanism. Another hypothesis is that rapid stellar rotation causes a preferred direction for mass ejection (Kwok \& Bignell 1984; Calvet \& Peimbert 1983; Morris 1981). This suggestion is, however, difficult to test. With the successful flight of the Infrared Astronomical Satellite (IRAS) and improved infrared, millimeter, and submillimeter instrumentation, proto-planetary nebulae (PPNe) have become the subject of much recent discussion (e.g., Volk \& Kwok 1989; Knapp et al. 1990).

Of particular interest is the highly evolved object AFGL 618. First identified as a bipolar nebula by Westbrook et al. (1975),

\footnotetext{
${ }^{1}$ Postal address: NRAO, Campus Building 65, 949 North Cherry Avenue, Tucson, AZ 85721.
}

it has been examined in detail from visible to radio wavelengths. The bright, compact $(\sim 0$ " 4 in diameter) infrared source studied by Westbrook et al. (1975) lies approximately midway between two visible nebulosities. They found it to have an infrared (3.5-34 $\mu \mathrm{m}$ ) color temperature of $T_{c} \approx 275 \mathrm{~K}$ (see also Kleinmann et al. 1978 and Deutsch et al. 1992). The bipolar structures are separated by $\sim 7^{\prime \prime}$ in the east-west direction (Calvet \& Cohen 1978). Centimeter-wavelength radio observations reveal an elongated compact source $(0,4 \times 0$."1) aligned with the bipolar lobes (Kwok \& Bignell 1984). Visible spectropolarimetry shows the two lobes to be reflection nebulae (Schmidt \& Cohen 1981; hereafter SC81). SC81 have derived an uncertain distance of $D \sim 1.8 \mathrm{kpc}$ (see discussion in $\S \mathrm{II} b$ of SC81). The total luminosity derived for this distance, $L_{\text {tot }} \sim 2.6 \times 10^{4} L_{\odot}$, is consistent with that expected for a postAGB star. The infrared spectrum is dominated by dust continuum emission, which arises primarily from the compact source (Kleinmann et al. 1978; Russell, Soifer, \& Willner 1978). CO observations as reported by Lo \& Bechis (1976), Thronson \& Mozurkewich (1983), Knapp \& Morris (1985), and Bachiller et al. (1988) indicate an expansion velocity for the outer envelope of about $V \approx 20 \mathrm{~km} \mathrm{~s}^{-1}$ and a mass-loss rate on the order of $\dot{M} \sim 10^{-4} M_{\odot} \mathrm{yr}^{-1}$.

Recent observations of the CO $(J=2 \rightarrow 1$ and $J=3 \rightarrow 2)$ rotational transitions (Gammie et al. 1989; Cernicharo et al. 1989 ) indicate outflow velocities $V>190 \mathrm{~km} \mathrm{~s}^{-1}$. In addition, there exist an expanding central $\mathrm{H}$ il region (Kwok \& Feldman 1981; Kwok \& Bignell 1984; Martín-Pintado et al. 1988) and 
high-velocity $\mathrm{H}_{2} v=1 \rightarrow 0 \mathrm{~S}(1)$ emission (Burton \& Geballe 1986). The ionization level and forbidden line emission from the visible lobes might be explained by clumpiness within the visible lobes or by low-velocity shocks (see SC81). From visible spectroscopy, Carsenty \& Solf (1982) derive an expansion velocity of the lobe material $V \sim 80 \mathrm{~km} \mathrm{~s}^{-1}$. The low-velocity component appears to be the remnant AGB circumstellar envelope, while the high-velocity component may reflect the onset of a "fast wind." Indeed, AFGL 618 has visibly brightened on a human time scale, increasing $\sim 2$ mag since 1940 (Gottlieb \& Liller 1976).

In this paper, we present $H$ - and $K$-band images and nearinfrared spectroscopy in the wavelength range $0.90-1.34 \mu \mathrm{m}$ of AFGL 618. The combination of an ultraviolet source and a molecular envelope in close proximity is expected to be favorable for fluorescent excitation of $\mathrm{H}_{2}$. This hypothesis will be tested by detailed modeling of the spectrum in $\S 4.2$. Earlier observations of molecular hydrogen emission (e.g., Thronson 1981; Beckwith, Beck, \& Gatley 1984; Burton \& Geballe 1986) have provided insight into the physical conditions in the nebula. We will discuss how the new observations strengthen or alter the earlier work. Section 5 includes a discussion of the IR images and presents models for the emission. In the following section, we describe the observations and present the data.

\section{OBSERVATIONS}

\subsection{Spectroscopy}

Observations of AFGL 618 were obtained on 1989 February 25-26, September 18-19, and November 15 through use of the Steward Observatory $2.3 \mathrm{~m}$ telescope and a new germanium diode spectrometer. The observations were at a resolving power of $\lambda / \Delta \lambda=1600$ at $1.164 \mu \mathrm{m}$. This instrument acquires simultaneous sky and object spectra, greatly speeding up the observing sequence and improving sky monitoring. A 6" circular aperture was used, thus taking in the majority of the eastern lobe of AFGL 618. Observations and data reduction were as described by Rix et al. (1990). The nearby G0 V star HR 1489 (Hoffleit \& Jaschek 1982) was used for flat-fielding and flux calibration (Campins, Rieke, \& Lebofsky 1985). Error in absolute flux calibration is estimated to be $\sim 15 \%$. The spectrum is presented in Figure 1. The rms noise level is typically $1 \mathrm{mJy}$; for reference, this is approximately twice the width of the line used to plot the spectrum.

\subsection{Imaging}

The Steward Observatory $64 \times 64$ pixel Rockwell HgCdTe array (Rieke, Rieke, \& Montgomery 1987) and $2.3 \mathrm{~m}$ telescope were used to obtain infrared images of AFGL 618 in standard $H$ - and $K$-bandpasses $\left(\lambda_{H}=1.60 \mu \mathrm{m}, \Delta \lambda_{H}=0.36 \mu \mathrm{m} ; \lambda_{K}=\right.$ $\left.2.2 \mu \mathrm{m}, \Delta \lambda_{K}=0.52 \mu \mathrm{m}\right)$. Observations were made on $1988 \mathrm{Sep}-$ tember 27 . The array was operated at $77 \mathrm{~K}$. Cold reimaging optics gave a scale of about 0.25 per pixel. The standard star HD 44612 (Elias et al. 1982) was observed for flux calibration. Sky flats were obtained after each exposure by wobbling the telescope $\sim 1^{\prime}$ to a region clear of infrared sources. Data reduction was carried out using standard IRAF routines with automated scripts provided by T. Greene. Seeing at the time and wavelength of the observations was $\sim 0$ ".7. The infrared images are displayed in the form of both linear and logarithmic contour plots in Figures 2 and 3. Integrated magnitudes are $H=11.59 \pm 0.1$ and $K=8.60 \pm 0.1$. We will discuss these images further in $\S 5$.

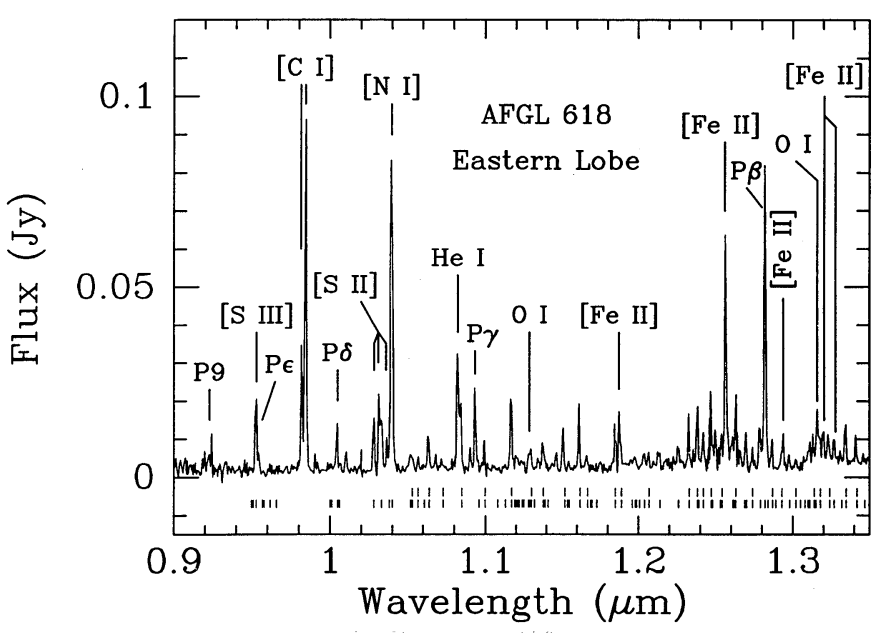

FIG. 1.-Near-intrared spectrum of AFGL 618 acquired with the Steward Observatory $2.3 \mathrm{~m}$ telescope and $\mathrm{Ge}$ spectrometer. Prominent atomic lines are labeled. Many of the weaker lines can be identified with molecular hydrogen vibration-rotation transitions. These are indicated by tickmarks below the spectrum. The upper set of tickmarks is for transitions which would be expected from thermally excited $\mathrm{H}_{2}$ at $T_{\mathrm{ex}} \approx 2000 \mathrm{~K}$. The lower set includes a fluorescent contribution (see $\S 4.2$ ). Based on a typical rms noise value of $\sim 1.0$ $\mathrm{mJy}$, it can be shown that parts of the spectrum are confusion-limited in atomic and molecular lines.

\section{THE ATOMIC LINE SPECTRUM}

Atomic lines prominent in the near-infrared spectrum of AFGL 618 are noted in Figure 1. A number of features identified here have already been reported by Westbrook et al. (1975) in their near-infrared spectrum. These include [C I] at 9823 and $9849 \AA$, [S II] at 10287, 10320, 10338, and $10373 \AA$, and H I P $\delta$ at $10049 \AA$. SC81 have analyzed the visible spectrum through the wavelength range $3750-7050 \AA$ in detail. The atomic emission lines will be discussed at greater length elsewhere (Kelly, Latter, \& Rieke 1992). However, it is worthwhile to make a brief examination here.

As in the visible spectrum presented by SC81, the near-IR spectrum is dominated by forbidden line emission. Through an analysis of spectropolarimetric data, SC81 showed that most of the visible permitted line radiation observed from the bright lobes originates within the central region. The photons are then scattered into the line of sight by dust. The visible forbidden line radiation arises in the low-density lobes themselves. Without polarization measurements, we cannot judge whether the infrared lines arise in a similar manner. For the infrared forbidden lines, this assumption is reasonable. The origin of the permitted $O_{I}$ lines at $1.3165 \mu \mathrm{m}\left(3 p^{3} P-4 s^{3} S^{o}\right)$ and $1.1287 \mu \mathrm{m}\left(3 p^{3} P-3 d^{3} D^{o}\right)$, however, is somewhat uncertain. Our failure to detect the $3 p^{5} P-4 s^{5} S^{o}$ line at $1.1295 \mu \mathrm{m}$ and the marginal detection of the $3 p^{5} P-3 d^{5} D^{o}$ line at $9261 \AA$, compared to the observed strengths of the 1.3165 and $1.1287 \mu \mathrm{m}$ lines, indicate that these lines are not produced dominantly by recombination (see Kelly et al. 1992).

It is possible that the $\mathrm{O}_{\mathrm{I}}$ lines are excited by continuum radiation absorbed in the $2 p^{43} P-4 s^{3} S^{0} 1040 \AA$ and $2 p^{43} P$ $3 d^{3} D^{o} 1026 \AA$ resonance transitions, resulting in emission at $1.3165 \mu \mathrm{m}$ and $1.1287 \mu \mathrm{m}$, respectively. The radiative excitation of $\mathrm{O}$ I lines has been discussed previously by Grandi (1975). The O I $1026 \AA$ transition is accidentally coincident with $\mathrm{H}$ I $\mathrm{Ly} \beta$; therefore, excitation of $\mathrm{O}_{\mathrm{I}}$ through this line can be enhanced if $\operatorname{Ly} \beta$ emission adds to the local continuum 


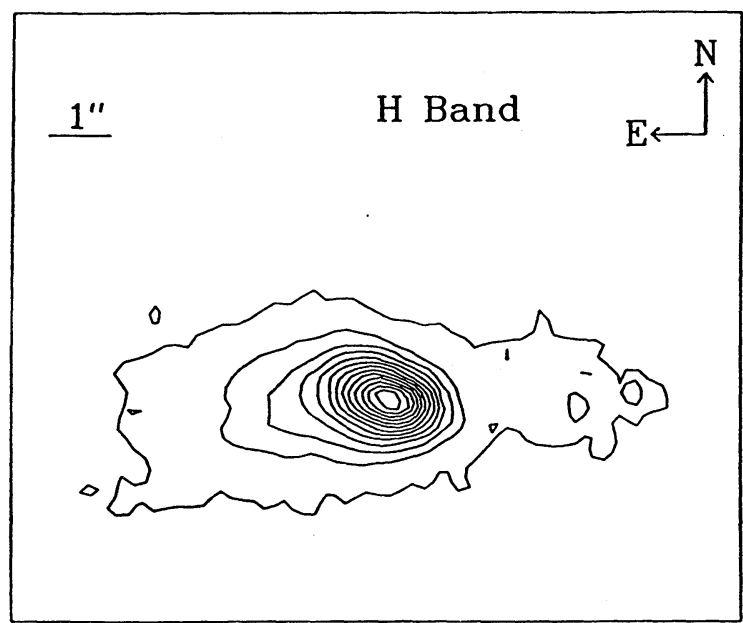

Fig. $2 a$

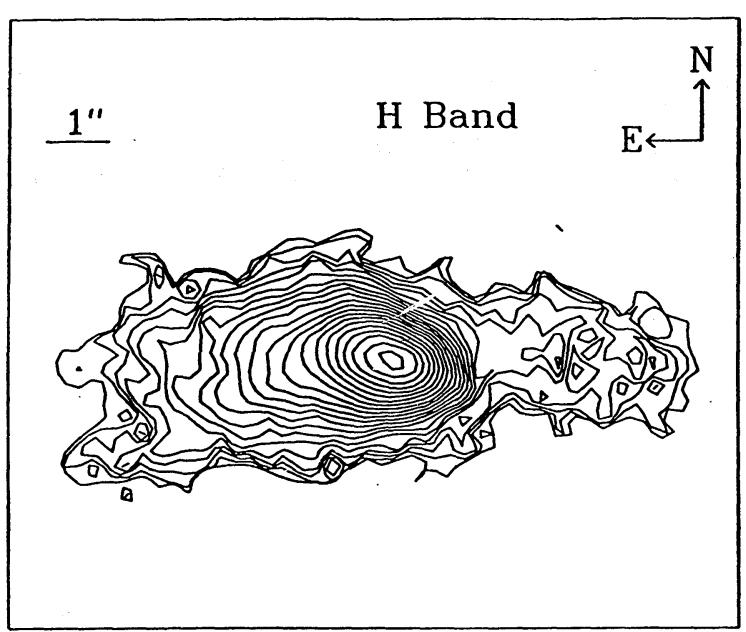

Fig. $2 b$

Fig. 2. - (a) $\mathrm{H}$-band image of AFGL 618 taken with the Steward Observatory $2.3 \mathrm{~m}$ telescope and $64 \times 64 \mathrm{HgCdTe}$ array. The scale is $0^{\prime \prime} 25$ pixel ${ }^{-1}$. Contours are at linear spacings with lowest at $1.9 \times 10^{-5} \mathrm{Jy} \mathrm{pixel}^{-1}$ and highest at $4.8 \times 10^{-4} \mathrm{Jy} \mathrm{pixel}^{-1}$. Contour spacings are $3.8 \times 10^{-5} \mathrm{Jy}_{\text {pixel }}{ }^{-1}$. (b) Same as Fig. $2 a$, but plotted with logarithmic contour spacing in order to display the lowest flux levels with greater clarity. The contour spacing is 0.08 dex with the lowest contour at $1.2 \times 10^{-5} \mathrm{Jy} \mathrm{pixel}^{-1}$ and highest at $4.8 \times 10^{-4} \mathrm{Jy} \mathrm{pixel}^{-1}$.

(Rudy, Rossano, \& Puetter 1989) and can be suppressed if there is strong $\operatorname{Ly} \beta$ absorption in the exciting stellar spectrum or by gas in the $\mathrm{O}_{\mathrm{I}}$ emitting region. The $1.1287 \mu \mathrm{m}$ line appears clearly detected; it is weaker than predicted by the continuum pumping model with no $\operatorname{Ly} \beta$ absorption, indicating that $\operatorname{Ly} \beta$ absorption is probably important. Another line of $\mathrm{O}_{\mathrm{I}}$ at $8446 \AA$ is expected to result from radiative excitation by the $1026 \AA$ and $1040 \AA$ continuum. This line was not identified by Westbrook at al. (1975), which means that if UV fluorescence of $\mathrm{O}_{\mathrm{I}}$ is important in AFGL 618 then $\operatorname{Ly} \beta$ absorption is large. Kelly et al. (1992) have detected the $8446 \AA$ line at a level which confirms that Ly $\beta$ absorption must depress the $1026 \AA$ continuum flux. The $\mathrm{O}_{\mathrm{I}}$ lines thus indicate that UV fluorescence is operative in this object; this will also be found from the molecular hydrogen emission (§ 4.2).

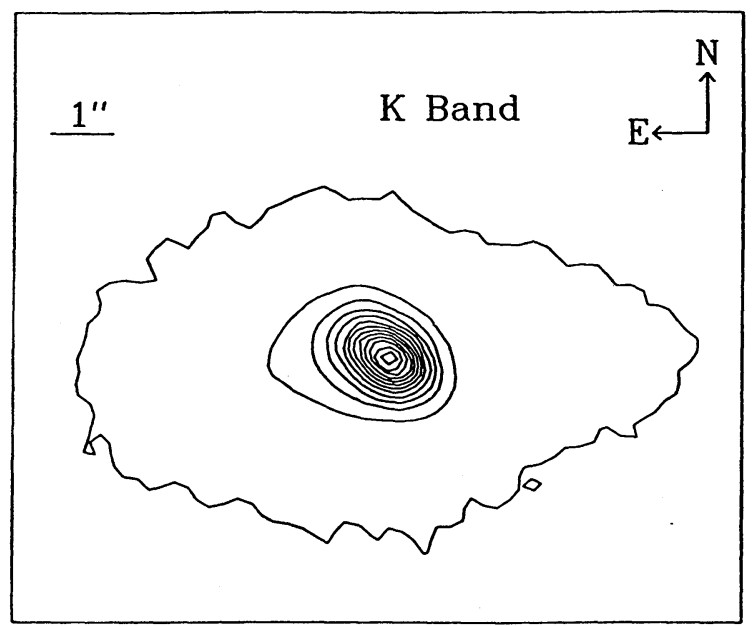

FIG. $3 a$

\section{THE MOLECULAR HYDROGEN EMISSION}

In our spectrum of AFGL 618, many features can be identified with quadrupole vibration-rotation transitions in the ground electronic state of $\mathrm{H}_{2}$. These are indicated by tickmarks below the spectrum in Figure 1. Indeed, most of the structure seen longward of $1.1 \mu \mathrm{m}$ is caused by $\mathrm{H}_{2}$ line emission, and in some regions the spectrum appears confusionlimited in $\mathrm{H}_{2}$ lines.

\subsection{Extinction to the $\mathrm{H}_{2}$-Emitting Region}

Observations of millimeter-wave emission from a number of molecular species (e.g., Thronson \& Mozurkewich 1983; Bachiller et al. 1988; Bujarrabal et al. 1988) demonstrate that a great deal of material surrounds AFGL 618, with an expansion

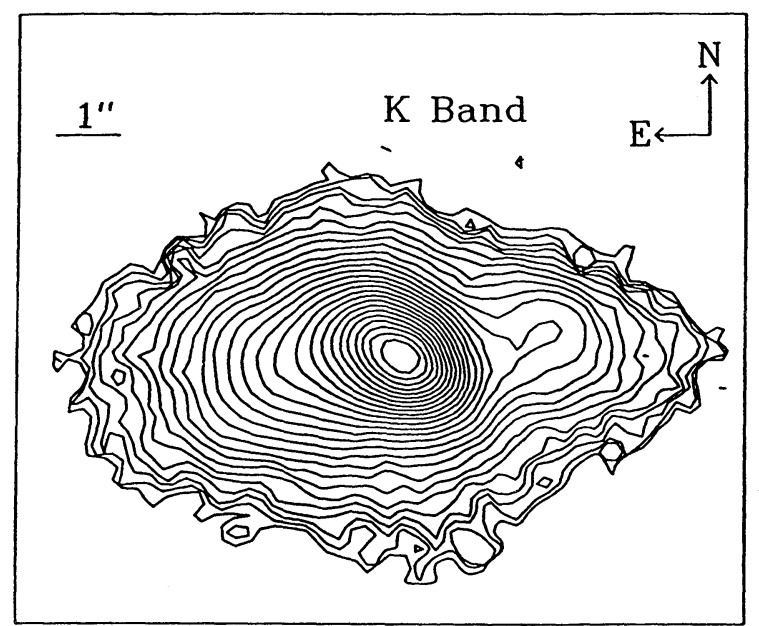

Fig. $3 b$

Fig. 3. - (a) $\mathrm{K}$-band image of AFGL 618 taken with the Steward Observatory $2.3 \mathrm{~m}$ telescope and $64 \times 64 \mathrm{HgCdTe}$ array. The scale is $0^{\prime \prime}$. 25 pixel ${ }^{-1}$. Contours are at linear spacings with lowest at $3.0 \times 10^{-5} \mathrm{Jy} \mathrm{pixel}^{-1}$ and highest at $7.2 \times 10^{-3} \mathrm{Jy} \mathrm{pixel}^{-1}$. Contour spacings are $6.0 \times 10^{-4} \mathrm{Jy}$ pixel ${ }^{-1}$. (b) Same as Fig. $3 a$; but plotted with logarithmic contour spacing in order to display the lowest flux levels with greater clarity. The contour spacing is 0.10 dex with the lowest contour at $1.9 \times 10^{-5} \mathrm{Jy} \mathrm{pixel}^{-1}$ and highest at $6.0 \times 10^{-3} \mathrm{Jy} \mathrm{pixel}^{-1}$. 
velocity consistent with it being the remnant mass-loss shell (Knapp \& Morris 1985; Bachiller et al. 1988). This material would have been ejected from AFGL 618 while it was on the AGB and prior to its evolution to a bipolar nebula. As a result, a large amount of attenuation due to dust in the extended circumnebular material is expected. However, a standard interstellar extinction law is probably inappropriate for an object like a PPN, if the bulk of the extinction is circumstellar rather than interstellar. Unfortunately, it is currently impossible to assess how much the true circumstellar extinction law might differ from that determined for the general interstellar medium. There are indications that grains formed in carbon-rich circumstellar envelopes are both smaller and more amorphous than grains found in the general interstellar medium (Martin \& Rogers 1987; Thronson et al. 1987; Rowan-Robinson \& Harris 1983).

Thronson (1981) used a standard interstellar extinction law and four pairs of $\mathrm{H}_{2}$ lines in the wavelength range 1.9-2.5 $\mu \mathrm{m}$ to determine a visual extinction of $A_{V}=8 \pm 6 \mathrm{mag}$ toward the $\mathrm{H}_{2}$-emitting lobes. The observations presented here provide a longer spectral baseline over which to determine the amount of attenuation than was available to Thronson. In the spectral region covered by the $\mathrm{Ge}$ spectrometer, there are eight $v=2 \rightarrow 0$ lines which share the same upper states with the four $v=2 \rightarrow 1$ lines present in Thronson's spectrum. Comparison of observed and predicted intensity ratios from these emission lines provides a measure of the attenuation. These eight $v=2 \rightarrow 0$ lines and measured peak flux densities are listed in Table 1 . Listed in Table 2 are the $v=2 \rightarrow 0$ and $v=2 \rightarrow 1$ lines which arise from the same upper state. For lines originating from the same upper state, the predicted line ratio is just the ratio of the products $\Delta E_{u l} A_{u l}$, where $\Delta E_{u l}$ is the energy of transition $u \rightarrow l$ and $A_{u l}$ is the spontaneous transition probability. Our best estimate using a standard interstellar extinction law (Rieke \& Lebofsky 1985) is $A_{V}=3.4_{-2.0}^{+4.0} \mathrm{mag}$. The relatively large uncertainty is a result of scatter in the extinction as determined from single line pairs, uncertainty in individual line fluxes, uncertainty in relative flux calibration for the two data sets, and blending with atomic and $\mathrm{H}_{2}$ fluorescence emission lines. This value for $A_{V}$ is well within the errors on $A_{V}$ as found by Thronson. There is also an uncertainty in $A_{V}$ that cannot be estimated, associated with the form of the extinction law. A differential extinction between 1.2 and $2.2 \mu \mathrm{m}$ of $A_{\lambda 1.2}-A_{\lambda 2.2}=0.62_{-0.36}^{+0.73} \mathrm{mag}$ is determined from the observations. However, there is a small dependence on the chosen extinction law in this estimate of the differential extinction. We

TABLE 1

\begin{tabular}{|c|c|c|}
\hline \multicolumn{3}{|c|}{$\mathrm{H}_{2}$ LiNe FluX Densities ${ }^{\mathrm{a}}$} \\
\hline$v=2 \rightarrow 0$ & $\lambda(\mu \mathrm{m})$ & $f_{v}^{v=2 \rightarrow 0}(\mathrm{Jy})$ \\
\hline$S(0)$ & 1.189 & $0.006 \pm 0.001$ \\
\hline$S(1)$. & 1.162 & $0.017 \pm 0.002$ \\
\hline$S(2)$. & 1.138 & $0.008 \pm 0.001$ \\
\hline$S(3)$. & 1.117 & $0.018 \pm 0.001$ \\
\hline$Q(5)$. & 1.263 & $0.017 \pm 0.002$ \\
\hline$Q(4)$ & 1.254 & $0.006 \pm 0.005$ \\
\hline$Q(3)$. & 1.247 & $0.017 \pm 0.004$ \\
\hline$Q(2) .$. & 1.242 & $0.008 \pm 0.001$ \\
\hline
\end{tabular}

${ }^{\text {a }}$ Uncertainties given are $1 \sigma$ statistical error in data only. Systematic uncertainty in flux calibration is estimated to be $\sim \pm 15 \%$.
TABLE 2

$\mathrm{H}_{2} v=2 \rightarrow 0$ AND $v=2 \rightarrow 1$ LINES ORIGINATING FROM THE SAME UPPER STATE ${ }^{\mathrm{a}}$

\begin{tabular}{|c|c|c|c|c|}
\hline$v=2 \rightarrow 0$ & $\lambda(\mu \mathrm{m})$ & & $v=2 \rightarrow 1$ & $\lambda(\mu \mathrm{m})$ \\
\hline$S(0)$ & 1.189 & $\leftrightarrow$ & $S(0)$. & 2.355 \\
\hline$S(1)$. & 1.162 & $\leftrightarrow$ & $S(1)$. & 2.247 \\
\hline$S(2)$. & 1.138 & $\leftrightarrow$ & $S(2)$. & 2.154 \\
\hline$S(3) .$. & 1.117 & $\leftrightarrow$ & $S(3)$. & 2.073 \\
\hline$Q(5)$ & 1.263 & $\leftrightarrow$ & $S(3)$. & 2.073 \\
\hline$Q(4) \ldots \ldots \ldots$ & 1.254 & $\leftrightarrow$ & $S(2)$. & 2.154 \\
\hline$Q(3) \ldots \ldots \ldots$ & 1.247 & $\leftrightarrow$ & $S(1) .$. & 2.247 \\
\hline $\mathscr{Q}(2) \ldots \ldots \ldots$ & 1.242 & $\leftrightarrow$ & $S(0) \ldots$ & 2.355 \\
\hline
\end{tabular}

${ }^{a}$ Data for $\mathrm{H}_{2} v=2 \rightarrow 1$ line fluxes are taken from Thronson $(1981$; see text).

also note that a best-fit $A_{V}$ as determined by $\mathrm{H}_{2}$ spectral models (see $\S 4.2$ ) is found to be $A_{V} \sim 3.0 \mathrm{mag}$. The position angle of polarization in the forbidden lines is nearly parallel to the Galactic plane (SC81), which would suggest that the extinction is interstellar in origin. In fact, the derived value of $A_{V}$ is consistent with the attenuation being interstellar, rather than intrinsic. The use of a standard interstellar extinction law would thus seem to be appropriate.

\subsection{Origin of the $\mathrm{H}_{2}$ Spectrum}

As demonstrated by Black \& van Dishoeck (1987), reflection nebulae are very likely to be sources of fluorescent $\mathrm{H}_{2}$ emission. Thus, AFGL 618 should be an excellent object in which to search for such emission. In addition, the spectral window accessible to the Ge spectrometer includes a large number of molecular hydrogen lines which will be pumped by fluorescence, making it possible to search for relatively low levels of fluorescent molecular hydrogen emission. Ultraviolet photons with $\lambda>912 \AA$ can escape the ionized nebula and illuminate the lobes where they can be absorbed in the Lyman $\left(B^{1} \Sigma_{u}^{+}-X\right.$ $\left.{ }^{1} \Sigma_{g}^{+}\right)$and Werner $\left(C^{1} \Pi_{u}-X^{1} \Sigma_{g}^{+}\right)$bands of molecular hydrogen. Approximately $10 \%$ of the resulting downward transitions will be to the vibrational continuum of the ground electronic state, thus leading to dissociation of the molecule (this mechanism was first suggested by Solomon in 1965 and discussed by Field, Somerville, \& Dressler 1966). The remaining transitions to bound vibration-rotation levels of the ground state result in UV line fluorescence and produce a distinctive infrared spectrum of slow electric quadrupole transitions. This process is described in detail by Black \& Dalgarno (1976), van Dishoeck \& Black (1986), Black \& van Dishoeck (1987), and Sternberg \& Dalgarno (1989).

Emission from low-lying $\mathrm{H}_{2}$ vibration-rotation levels can be collisionally excited in regions with kinetic temperature $T_{K} \gtrsim$ $1000 \mathrm{~K}$. From the relatively weak $v=2 \rightarrow 1 S$ lines near $2 \mu \mathrm{m}$, Thronson (1981) has argued that much of the $\mathrm{H}_{2}$ emission from AFGL 618 is collisionally excited in shocks (see also Beckwith et al. 1984). An alternative hypothesis has been suggested by Jura \& Kroto (1990) to explain the $\mathrm{H}_{2}$ emission from AFGL 2688 (the "Egg Nebula"). In their model, $\mathrm{H}_{2}$ is collisionally excited in hot gas which is created by a rapid grain streaming velocity.

We have attempted to reproduce the observed $\mathrm{H}_{2}$ emission from AFGL 618 using the modeling techniques described by van Dishoeck \& Black (1986) and Black \& van Dishoeck (1987). Both fluorescent emission spectral models and models assuming thermal equilibrium have been calculated. In their 
fluorescence models, $\mathrm{H}_{2}$ is formed primarily on grain surfaces and destroyed by the process of spontaneous radiative dissociation as described above. The abundance of $\mathrm{H}_{2}$ is assumed to be in steady state. The calculation solves the equation of transfer for 22,445 absorption lines simultaneously in a planeparallel cloud illuminated on one side by an external ultraviolet radiation source. This type of simple geometry is not intended to reproduce the actual distribution of material in AFGL 618. It should, however, produce results which are good averages of the conditions actually present. Also, such models can aid in line identification and verify the presence of fluorescent emission in addition to providing an estimate of the level of excitation by UV photons. Statistical equilibrium equations are solved for the populations of 211 vibration-rotation levels in the $X^{1} \Sigma_{g}^{+}$state, 629 levels in the $B^{1} \Sigma_{u}^{+}$state, and 476 levels in the $C^{1} \Pi_{u}$ state. Multiple steps are taken through the cloud at each of which the cascade of 2937 vibration-rotation transitions in the $X^{1} \Sigma_{g}^{+}$state is calculated.

The intensity of the radiation field at the molecular boundary is parameterized by the factor $I_{\mathrm{UV}}$, such that $\phi=4.5$ $\times 10^{-8} I_{\text {Uv }}$ photons $\mathrm{cm}^{-2} \mathrm{~s}^{-1} \mathrm{~Hz}^{-1}$ at $\lambda=1000 \AA$. In regions where the Ly $\alpha$ flux is large, enhanced $\mathrm{H}_{2}$ excitation might occur due to accidental resonances with the $B^{1} \Sigma_{u}^{+}-X^{1} \Sigma_{g}^{+}$ $v=1-2 P(5)$ and $R(6)$ transitions, provided that $v=2$ level in the $X^{1} \Sigma_{g}^{+}$ground electronic state is already significantly populated. To explore this possibility in AFGL 618, we have examined the effect of a large Ly $\alpha$ flux on the IR fluorescence spectrum.

Thronson (1981) determined an $\mathrm{H}_{2}$ vibration-rotation excitation temperature based on line fluxes in the $2 \mu \mathrm{m}$ region that were not corrected for extinction. To minimize error in the thermal model of $\mathrm{H}_{2}$ emission, we have corrected the fluxes presented by Thronson for $A_{V}=3.0 \mathrm{mag}$ and a standard extinction law. We have determined a new excitation temperature based on the corrected fluxes, even though the change was expected to be small. Assuming a Boltzmann distribution and including all 15 available lines in a least-squares fit to a plot of $N_{u} / g_{u}$ versus $T_{u}=E_{u} / k$ (see, e.g., Thronson 1981), we found that $T_{\mathrm{ex}}=2250 \mathrm{~K}$. However, as was also shown by Thronson, the four $v=2 \rightarrow 1$ lines all appear to be systematically stronger than would be expected for thermal equilibrium, based on the $v=1 \rightarrow 0$ lines. Since these are also the weakest lines in the spectrum, this might be a result of measurement error. In addition, scatter in the $N_{u} / g_{u}$ versus $T_{u}$ diagram is much larger in the $v=2 \rightarrow 1$ lines. It therefore seems prudent to leave these lines out of the fit (as was also done by Thronson) and determine a $T_{\text {ex }}$ based only on the $v=1 \rightarrow 0$ lines. For such a least-squares fit, it was found that $T_{\mathrm{ex}}=1942$ $\pm 200 \mathrm{~K}$. This can be compared to the value found by Thronson of $T_{\mathrm{ex}}=1950 \pm 200 \mathrm{~K}$. The difference between the two estimates is completely negligible.

Results of the modeling are presented in Figure 4. A thermal model appears on the bottom, and a fluorescent model is displayed directly below the data. Both models extend into the $2 \mu \mathrm{m}$ region, and have been adjusted to give the same intensity in the $v=1 \rightarrow 0 S(1)$ line as that given by Thronson (1981). This results in an $\mathrm{H}_{2}$ column density of $N_{\mathrm{H}_{2}}=5.2 \times 10^{18}$ $\mathrm{cm}^{-2}$ for $T_{K}=2000 \mathrm{~K}$. Characteristics of the spectrum are unchanged if $T_{K}=1942 \mathrm{~K}$. The fluorescent model has been calculated for $I_{\mathrm{UV}}=200, n_{\mathrm{H}}=10^{5}$, and $T_{K}=2000 \mathrm{~K}$. This value of $I_{\mathrm{UV}}$ is for a UV radiation field appropriate to a B0 (SC) 1 ) star embedded in an $\overline{\mathrm{H}}$ II region, extinguished by about $A_{V} \sim 1.5-2.0 \mathrm{mag}$, and diluted over a distance of $r \approx 10^{17} \mathrm{~cm}$,

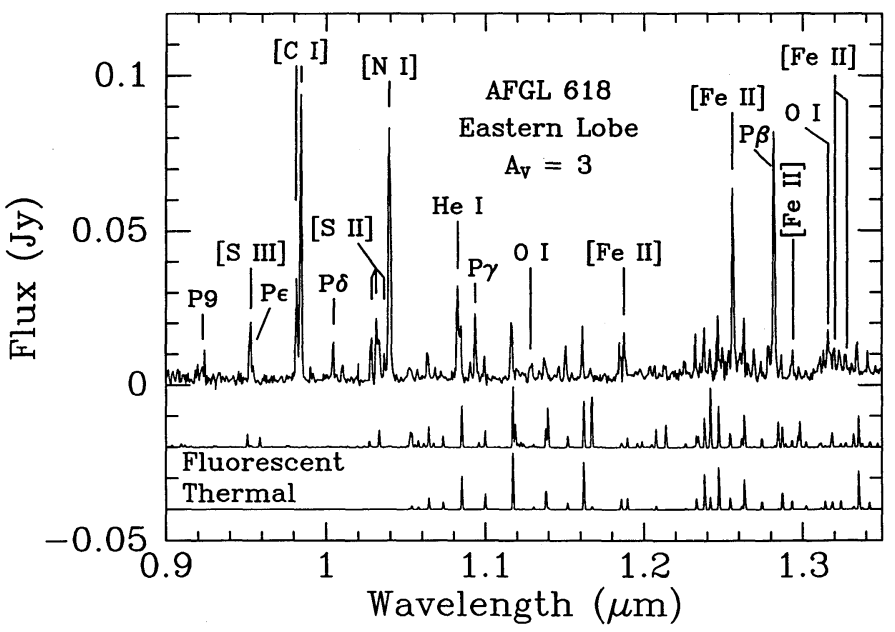

Fig. 4.-Spectrum plotted in Fig. 1 compared with spectral models of thermal and fluorescent emission from $\mathbf{H}_{2}$. The data appear on top, the fluorescence model in the middle, and the thermal model on the bottom. Parameters for the models are $T_{\mathrm{ex}}=2000 \mathrm{~K}$ and $N_{\mathrm{H}_{2}}=5.2 \times 10^{18} \mathrm{~cm}^{-2}$ (thermal), $I_{\mathrm{UV}}=200, n_{\mathrm{H}}=10^{5}$, and $T=2000 \mathrm{~K}$ (fluorescence; see text). The predicted fluxes have been corrected for $A_{V}=3.0 \mathrm{mag}$. The flux scale is referenced to the data.

which is comparable to the linear scale of the near-IR lobes in AFGL 618. Both models include $A_{V}=3.0 \mathrm{mag}$ along the observer's line of sight. (We are thus assuming that the extinction to the lobes derived in $\S 4.1$ is entirely interstellar.) It is important to note that these sources of attenuation are separate from the attenuation internal to the lobe, which is calculated using a total gas column density to visual extinction of $N_{\text {tot }} / A_{V}=6.36 \times 10^{20} \mathrm{~cm}^{-2} \mathrm{mag}^{-1}$. This is consistent with the gas-to-dust ratio for carbon stars as found by Jura (1986).

A satisfactory fit to the strongest emission lines is produced by the thermal model alone, but the strengths of the weaker molecular hydrogen lines require a fluorescent component. At least four features appear stronger in the data than predicted by either model. These lines include $v=3 \rightarrow 1 S(5)(1.152 \mu \mathrm{m})$, $3 \rightarrow 1 S(3)(1.185 \mu \mathrm{m}), 3 \rightarrow 1 S(1)(1.233 \mu \mathrm{m})$, and $2 \rightarrow 0 Q(3)$ $(1.247 \mu \mathrm{m})$. The last two are in a region crowded by other lines. Several models were calculated in which the value of $I_{\mathrm{UV}}$ was varied over many orders of magnitude. A better fit than that presented was not obtained. It is possible that these lines are confused with unidentified atomic and/or molecular features. Also, the plane-parallel geometry used in the models might be an inadequate representation of AFGL 618.

In addition, it is important to note that at least three lines are predicted to be stronger by the fluorescence model than observed in the data. These are the $v=3 \rightarrow 1 S(6)(1.139 \mu \mathrm{m})$, $3 \rightarrow 1 S(4)(1.167 \mu \mathrm{m})$, and $4 \rightarrow 2 S(4)(1.242 \mu \mathrm{m})$ lines. Comparison with a model calculated for a large Ly $\alpha$ flux suggests that these lines can be enhanced by Ly $\alpha$ pumping. Therefore, it would appear that the Ly $\alpha$ flux used in the model shown is too large. The value chosen was based on a simple estimate of the leakage of $\mathrm{Ly} \alpha$ photons from the $\mathrm{H}$ II region and could easily be an overestimate for a region where dust competes effectively with resonant scattering. A full range in $L y \alpha$ flux was not explored, however.

While the model fit might not be exact, it is important to note that everywhere a feature is predicted, one is seen. In some cases the detection is marginal, but no anticoincidences are found. If we assume that the discrepancies between the model results and the data are due to the complicated geometry 
actually present in AFGL 618, we can use them to infer details about the morphology within the bipolar lobes. Most importantly, it appears that $I_{U V}$ is not uniform throughout the lobes. Possible variations in total density should also be taken into account.

\section{THE IR IMAGES}

Our infrared images clearly show AFGL 618 to be extended in the east-west direction (Figs. 2 and 3), with a western lobe that is much fainter than its eastern counterpart. This appearance is in harmony with the bipolar morphology that has been reported previously. The emission at both $H$ and $K$ is dominated by a small, unresolved source whose projected position is just inside the eastern lobe. The position is consistent with the placement of the peak $2.1 \mu \mathrm{m}$ continuum as found by Beckwith et al. (1984). This object has previously been considered to be a warm dust torus which surrounds the central star and $\mathrm{H}$ II region (Westbrook et al. 1975; Thronson 1981). However, as will be discussed, this near-IR peak is not likely to have the same origin nor to be at precisely the same location as the peak infrared emission at wavelengths $\gtrsim 3 \mu \mathrm{m}$.

Westbrook et al. (1975) carried out single-aperture photometry of AFGL 618, and found $H=11.6 \pm 0.1 \mathrm{mag}$ and $K=9.3 \pm 0.1 \mathrm{mag}$. Our value of $K=8.60 \pm 0.1 \mathrm{mag}$ is clearly in disagreement with the Westbrook et al. (1975) result. This can be understood if we consider that the flux at $B$ has been

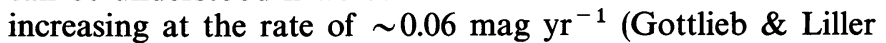
1976). We will see below that most of the flux at $K$ is due to light from the central object scattered into the line of sight, as is also the case at visual wavelengths (SC81). Therefore, we might expect the $K$ brightness to increase at a similar rate. However, our agreement with Westbrook et al. (1975) at $H$ is good, where a similar brightening should have occurred. On the other hand, the integrated fluxes are dominated by the central component. The spectral energy distribution of the central source shows that warm dust contributes significantly at $K$ and not at $H$ (Westbrook et al. 1975). Therefore, a slight temperature change in the hottest dust might cause the source to brighten at $K$ but not at $H$. In this scenario, the $B$-band brightening observed by Gottlieb \& Liller (1976) must have ceased since the observa- tions of Westbrook et al. (1975). We suggest that continued monitoring at IR and visible wavelengths would be valuable.

The morphology of AFGL 618 in these images is consistent with a substantial inclination of the bipolar axis to the line of sight, such that the eastern lobe lies nearest the Earth. Carsenty \& Solf (1982) determined just such an orientation from an analysis of the radial velocity structure. They found the polar axis to be inclined to the plane of the sky at an angle of $i \sim 45^{\circ}$. This type of viewing geometry can account for the position of the compact source, and at least in part, the smaller apparent size of the western lobe as seen in these and visible images.

To better understand the morphology of the IR images, we have calculated models of the emission. Assuming that most of the observed emission is due to scattered light from the compact object, we have employed the semi-analytic singlescattering formulae of Morris (1981). Yusef-Zadeh, Morris, \& White (1984) have presented a detailed analysis of bipolar nebulae using Monte Carlo simulations of multiple scattering. Such an analysis is beyond the scope of this paper. However, comparisons of multiple scattering with single scattering models reveal that models of single scattering alone should produce good first-order results, because most of the observed emission is scattered in regions of low optical depth (see YusefZadeh et al. 1984).

The models assume isotropic scattering. The wavelengthdependence of optical depth (the constant $c$ in Morris' eq. [2]) is assumed to follow the Rieke \& Lebofsky (1985) extinction law. As we did not attempt to match the emitted flux (which depends on a large number of poorly determined parameters), the albedo has been taken to be unity. Such a large value of the albedo is of course unphysical; it should be kept in mind that for our purposes the albedo is simply a scaling factor. In scaling the $H$ - and $K$-band models for comparison, we have implicitly assumed that the albedo is the same at $H$ and $K$.

The results of the modeling exercise are shown in Figure 5 in the form of contour plots. A model of the $H$-band image (Fig. $5 a$ ) is plotted with lowest contour at a value 0.2 dex fainter than the model for the $K$-band image (Fig. $5 b$ ) to be consistent with the data (Figs. $2 b$ and $3 b$ ). The models have been convolved with a Gaussian of FWHM $=0$."7 to simulate the

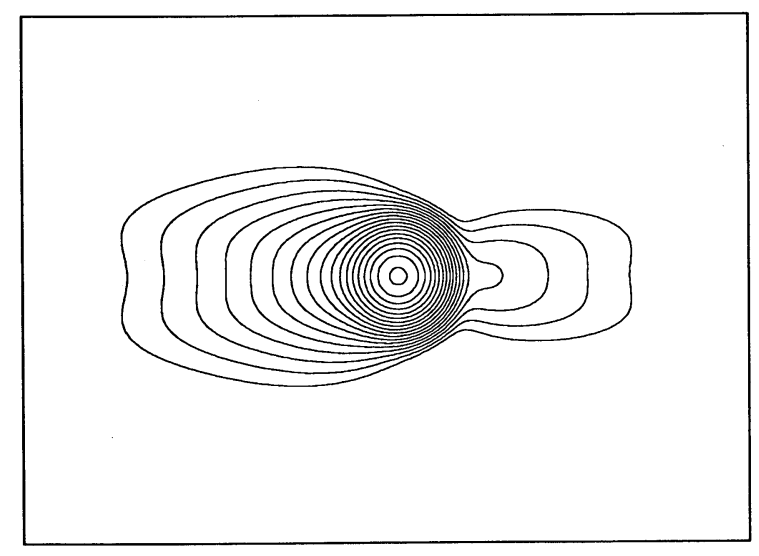

FIG. $5 a$

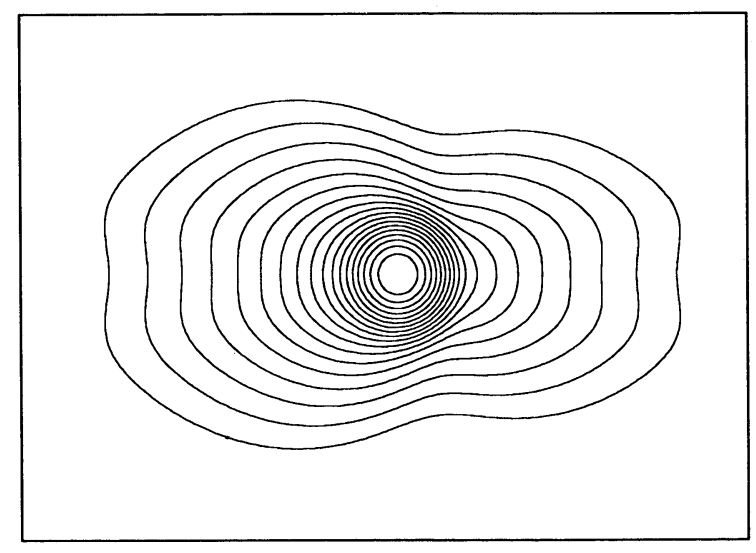

FIG. $5 b$

FIG. 5.-Single-scattering models of the infrared emission from AFGL 618 for the dust distribution shown in Fig. 6 and described in the text. The inclination to

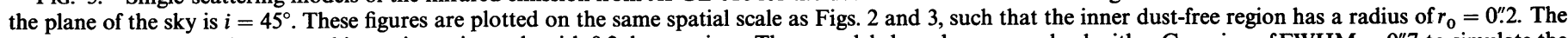

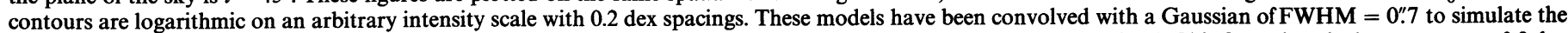

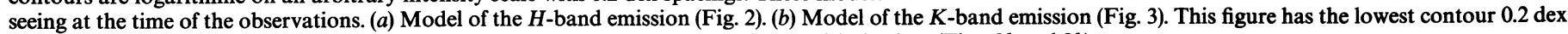
higher in brightness than the corresponding contour displayed in $(a)$ to be consistent with the data (Figs. $2 b$ and $3 b$ ). 


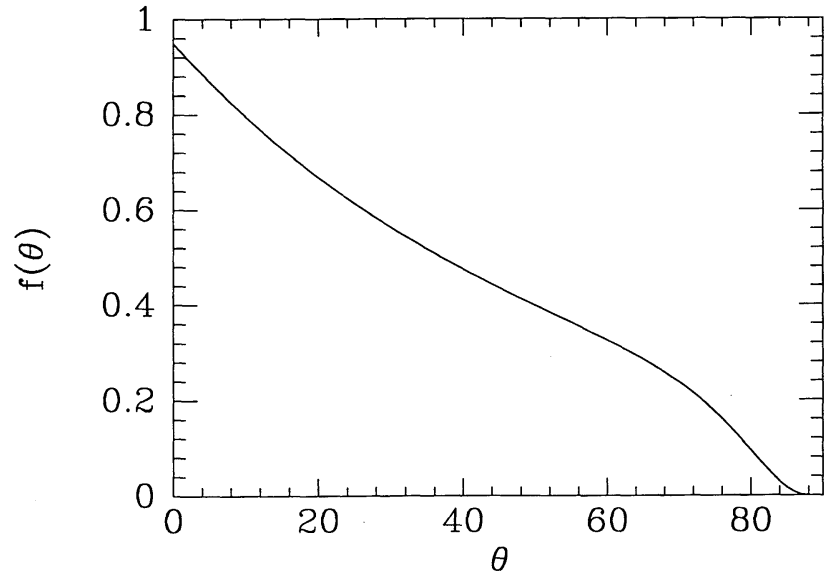

Fig. 6.-The dust distribution $f(\theta)$ used in the models presented in Fig. 5. The angle $\theta$ is the stellar latitude above the equator. There is zero density in dust above $\theta=88^{\circ}$. A functional form of this distribution is given in the text.

seeing at the time of the observations. Figure 6 displays the distribution of dust which was found to reproduce best the IR images. This distribution has the functional form (following Yusef-Zadeh et al. 1984)

$$
f(\theta)=\left(1-\sin \theta+\frac{1}{2} \sin ^{2} \theta-\frac{1}{6} \sin ^{3} \theta\right) C(\theta),
$$

where

$$
C(\theta)=\left[1+\left(\frac{\cos 75^{\circ}-\cos 88^{\circ}}{\cos \theta-\cos 88^{\circ}}\right)^{2}\right]^{-1} \quad \text { if }|\theta|<88^{\circ},
$$

and $C(\theta)=0$ if $|\theta| \geq 88^{\circ}$. Here $\theta$ is the stellar latitude such that $\theta=0$ at the equator. Note that this distribution is very much like that found by Yusef-Zadeh et al. (1984) for the similar object AFGL 2688. The inclination angle to the plane of the sky for the models shown is $i=45^{\circ}$. We have plotted the models on the same linear spatial scale as used in Figures 2 and 3. Included in the models is an inner dust-free region with a radius $r_{0}$ scaled in the figures so that $r_{0}=0$. 2 . The angular extent of this ionized region seems well established (e.g., Westbrook et al. 1975; Kwok \& Bignell 1984; Martín-Pintado et al. $1988)$. This gives an inner radius of $r_{0} \approx 5.4 \times 10^{15}(D / 1.8 \mathrm{kpc})$ $\mathrm{cm}$, or about 2.6 times smaller than the inner radius found by Jura \& Kroto (1990) for the apparently less evolved object AFGL 2688. The time scale for post-AGB evolution is extremely sensitive to the mass of the star, however (Iben \& Renzini 1983).

The optical depth at infinity has the functional form $\tau_{\lambda}(\theta)=$ $\tau_{\gamma}^{0} \chi f(\theta)$ where $\chi$ is a constant defined such that $\chi f(\theta=0)=1$. For the models displayed, we find that the optical depth at infinity along the stellar equator is $\tau_{H}(\theta=0)=15.2$ at $\lambda=1.65$ $\mu \mathrm{m}$ and $\tau_{K}(\theta=0)=9.1$ at $\lambda=2.2 \mu \mathrm{m}$. This translates, using a standard interstellar extinction law (Rieke \& Lebofsky 1985), to $A_{V} \approx 87 \mathrm{mag}$. This is in reasonable agreement with that expected from Jura's (1986) estimate of the dust mass-loss rate from AFGL 618; if we relate the optical depth to the mass-loss rate as in Jura's (1986) equation (5) and integrate over the dust angular distribution function, we find that the equatorial visual optical depth ranges from 34 to 140 for $r_{0}=(5.4-1.3)$ $\times 10^{15} \mathrm{~cm}$.

Note that the model images are more strongly peaked than the data. Also, while somewhat noisy, the western lobe in the $H$-band data appears slightly more extended than the model at $\lambda=1.65 \mu \mathrm{m}$. Both of these differences are probably caused by our neglect of multiple photon scattering (see Yusef-Zadeh et al. 1984). The unconvolved models have a very large gradient in intensity just to the west of the brightest contour. Smoothing has decreased this rapid rise to a level much more like that seen in the data. Because the contours have logarithmic spacing, smoothing had little effect on the appearance of the models away from the bright peak. In both images the bright region appears to be elongated in the northeast-southwest direction. This elongation is not reproduced in the models, and suggests that the actual distribution of dust is slightly asymmetric. Many of the details seem to be well modeled, however, such as the flattening of the contours in the polar regions as well as the relative extent of the two images. Because of the large optical depth at $\lambda=6500 \AA \AA$ implied by our IR models, no attempt was made to reproduce the visible light image displayed in Westbrook et al. (1975). This image is from the Palomar Red Sky Survey, and no other visible light image is known to the authors.

In scaling the models relative to one another, we have used the relative flux values at $H$ and $K$ as determined from our data. As mentioned above, our $K$-band flux is 1.9 times higher than that of earlier work. The models presented here have relative sizes scaled to the relative fluxes in our data. If the lowest model contours were scaled to a $K$-band flux that is reduced by a factor of 2 , the relative sizes of the models would no longer resemble the data. This suggests that our flux calibration is not greatly in error.

It seems worthwhile to note that the dust distribution shown for AFGL 2688 in Figure 2 of Yusef-Zadeh et al. (1984) (similar to our Fig. 6) is not that given by their caption. The function $f(\theta)$ described by the parameters given in their caption for AFGL 2688 produces single-scattering model images consistent with their Figure $4 a$; the distribution displayed in their Figure 2 does not.

\section{DISCUSSION}

The $\mathrm{H}_{2}(v=1 \rightarrow 0)$ lines in the $2 \mu \mathrm{m}$ region are rather intense and exhibit a rotational excitation temperature in the $v=1$ levels of $T_{\mathrm{ex}} \approx 2000 \mathrm{~K}$ (Thronson 1981). This immediately suggests an important component of high-temperature thermal emission, perhaps even in lines of the $v=2 \rightarrow 0$ band in the spectral region accessible to the Ge spectrometer $(\lambda \lesssim$ $1.6 \mu \mathrm{m})$. If the excitation temperature were $T_{\mathrm{ex}} \lesssim 2000 \mathrm{~K}$ for all levels, however, little emission would be expected in transitions arising in $v^{\prime} \geq 3$. The presence of such highly excited lines would reveal a component of fluorescent emission. We have used model spectra to aid the identification of $\mathrm{H}_{2}$ lines in the rich near-infrared spectrum. As discussed in $\S 4.2$, several $v=3 \rightarrow 1$ lines seem to be present: although weak, their intensities are higher than expected from thermal emission alone, indicating the presence of a fluorescent $\mathrm{H}_{2}$ component.

Fluorescent emission is expected from bright reflection nebulae like AFGL 618, and its detection can provide information about the detailed morphology of this object. Since fluorescent emission is only weakly present in the spectrum of AFGL 618, the UV flux incident on the lobes must be highly extinguished in the region surrounding the central star. Alternatively, the dust distribution may prevent the observed region from having a direct view of the stellar photosphere. Also, fluorescent emission could be strong within the interior of the lobe, but extinguished by the large amount of circumnebular material to the intensity found in the spectrum presented here. 
The presence of reflected stellar continuum in the visible spectrum (SC81) demonstrates that some stellar light reaches the lobes (see also $\S 5$ ). It is not possible with the current data to determine if the extinction to the fluorescent $\mathrm{H}_{2}$ differs from that to the thermal component, or if the fluorescent and thermally excited $\mathrm{H}_{2}$ are co-extensive. SC81 postulate the presence of optically thick knots close to the central star that shadow much of the lobe material from direct exposure to the stellar radiation field. Evolved forms of these clumps might be visible as knots in images of PNe (e.g., Balick 1989). The analysis of SC81 revealed that the unshielded material is ionized with an electron temperature of $T_{e} \approx 1.8 \times 10^{4} \mathrm{~K}$ and electron density of $n_{e} \approx n_{\mathrm{H}} \approx 5 \times 10^{4} \mathrm{~cm}^{-3}$. The shadowed component near the clumps is primarily neutral with $T_{e} \approx 10^{4} \mathrm{~K}, n_{e} \approx 10^{3}$ $\mathrm{cm}^{-3}$, and $n_{\mathrm{H}} \approx 10^{5} \mathrm{~cm}^{-3}$. Heating of the neutral component takes place through ionization of hydrogen by UV radiation reflected from the unshielded component. It is possible that UV-excited molecular hydrogen is present within the shadowed material, but farther out in the lobes where the gas is cool and molecules can easily survive. The fluorescence-producing UV photons could be delivered into the shielded region by scattering off dust in the material that is directly exposed to starlight.

Models of collisionally excited $\mathrm{H}_{2}$ emission were also compared to the data. These reproduce the most prominent features of the observed $\mathrm{H}_{2}$ spectrum adequately for a uniform excitation temperature of $T_{\mathrm{ex}} \sim 2000 \mathrm{~K}$, but a significantly better fit results when a component of fluorescent emission is included. Thronson (1981) and Burton \& Geballe (1986) have discussed in detail the origin of the thermal emission in AFGL 618. Thronson argued that the molecular hydrogen is excited by shocks with velocities of $\sim 10 \mathrm{~km} \mathrm{~s}^{-1}$. He found the total mass of shocked $\mathrm{H}_{2}$ to be $M_{\mathrm{H}_{2}} \sim 10^{-4} M_{\odot}$ if the object is at $1 \mathrm{kpc}$. Adopting the column density in our thermal models of $N_{\mathrm{H}_{2}}=5.2 \times 10^{18} \mathrm{~cm}^{-2}$ and assuming the emission arises from a spherical region with $r=5 \times 10^{16}(D / 1.8 \mathrm{kpc}) \mathrm{cm}$, we find the total mass of $\mathbf{H}_{2}$ to be $M_{\mathbf{H}_{2}} \sim 5 \times 10^{-5} M_{\odot}$. This assumes that the emitting $\mathrm{H}_{2}$ is uniformly distributed throughout the eastern lobe. If shocks are responsible, then the emission might be expected to arise from a relatively small region. It is not clear that this must be the case. Beckwith et al. (1984) have found the $\mathrm{H}_{2} v=1 \rightarrow 0 \mathrm{~S}(1)$ line emission to be extended. Spectra near $1.1 \mu \mathrm{m}$ of higher signal-to-noise ratio acquired through a small aperture at various points on the bright eastern lobe could be revealing.

In a clumpy medium, cooling of the gas within the shadows might generate low-velocity shocks of the type required to excite $\mathrm{H}_{2}$ emission. Burton \& Geballe (1986) suggest a similar model based on rapidly moving clumps. Shocks generated by the stellar mass-loss are probably also present. If there is a shock at the interface of the high-velocity wind and the slower moving envelope material, it will likely be strongly dissociating, and therefore will not contribute significantly to the total $\mathrm{H}_{2}$ emission. However, at least some of the $\mathrm{H}_{2}$ emission must come from material accelerated by the fast wind as evidenced by the very broad velocity width $\left(\sim 250 \mathrm{~km} \mathrm{~s}^{-1}\right)$ of the $v=1 \rightarrow 0 S(1)$ line (Burton \& Geballe 1986). It is possible that the high-velocity molecular material has reformed after being destroyed in a fast shock (e.g., Cernicharo et al. 1989). However, polyatomic species like $\mathrm{HCO}^{+}, \mathrm{HCN}$, and $\mathrm{HC}_{3} \mathrm{~N}$ have been observed by Cernicharo et al. (1989) also to be present at high velocities. The rapid expansion of the material will make reformation of such species highly unlikely if the gas is directly exposed to the bright central star. Hartquist \& Dyson (1987) have suggested a mechanism by which molecular material can be accelerated to high velocities without being dissociated in a fast shock. Also, Glassgold, Mamon, \& Huggins (1991) have shown that molecule formation in a fast wind can be efficient. However, the UV brightness of their model star is much weaker than that present in AFGL 618. It is impossible to determine how much the high-velocity material contributes to the emission measured in our data. Also, while shock excitation is sufficient to explain the observed emission, it might not be necessary. There are neutral regions within AFGL 618 which are at high enough density and temperature (SC81) to maintain $\mathrm{H}_{2}$ nearly in LTE at $T_{\text {ex }} \sim T_{K} \sim 2000 \mathrm{~K}$. The above mentioned model of heating by rapid grain streaming suggested by Jura \& Kroto (1990) could also be important.

Recently, Lequeux \& Jourdain de Muizon (1990) presented a measurement of the $\mathrm{H}_{2} v=1 \rightarrow 0 O(5)$ line $(3.234 \mu \mathrm{m})$ from AFGL 618. A total flux in the line of $f=8 \times 10^{-13} \mathrm{ergs} \mathrm{cm}^{-2}$ $\mathrm{s}^{-1}$ was found in a $5^{\prime \prime}$ aperture. Contrary to a statement made in their paper, this value is significantly larger than predicted by thermal or fluorescent excitation when compared to the $v=1 \rightarrow 0 S(1)$ line of Thronson (1981). Comparison with our models at $T_{K}=2000 \mathrm{~K}$ suggests that $A_{V} \approx 23 \mathrm{mag}$ is required to reproduce the measured $v=1 \rightarrow 0 O(5)$ line flux. Unless the true extinction law in AFGL 618 is extremely unusual, we must conclude that the discrepancy is due to differences in flux calibration.

On the basis of the Balmer decrement and visible line ratios, an extinction of $A_{V} \sim 3.5 \mathrm{mag}$ (Westbrook et al. 1975; Calvet \& Cohen 1978; SC81) has been found. We have determined that $A_{V}=3.4_{-2.0}^{+4.0} \mathrm{mag}$ to the $\mathrm{H}_{2}$-emitting region ( $\S 4.1$ ). Thus, the visible and infrared line radiation appear to be attenuated by approximately the same dust distribution. In contrast, Thronson (1981) found $A_{V} \gtrsim 28 \mathrm{mag}$ to the region that emits H I Bry (see also Lequeux \& Jourdain de Muizon 1990). As discussed by Thronson, the Br $\gamma$ emitting region is the compact $\mathrm{H}$ II region which lies behind a thick, dusty torus surrounding the central star, obscuring it from view at visible wavelengths. The intensity of fluorescent $\mathrm{H}_{2}$ emission implies uneven, or extinguished ultraviolet illumination of the visible lobes. Also, it might be incorrect to assume a Case B recombination spectrum for the intrinsic Balmer decrement in a compact, very dense nebula (see Thompson 1987, and references therein). The problem is compounded if a nonstandard extinction law applies. The distributions of attenuating and emitting gas are complex and still not fully delineated.

Our analysis of the infrared images supports the basic morphology of bipolar nebulae suggested by Morris (1981) and Yusef-Zadeh et al. (1984). Therefore, the bright compact source which dominates the flux at $\lambda \lesssim 2 \mu \mathrm{m}$ is not due to dust emission from a dense torus, but is a region where a relatively large number of scattered photons can escape. Similar regions are observed and have been modeled in other sources with much smaller inclination angles, like AFGL 2688 (Morris 1981; Yusef-Zadeh et al. 1984). In such objects the brightening is seen well away from the position of the central star. The near coincidence of the bright source with the position of the central star in AFGL 618 is due to a relatively large inclination angle. Infrared images at wavelengths $8.3 \mu \mathrm{m} \leq \lambda \leq 12.4 \mu \mathrm{m}$ acquired by Deutsch (1990) and Deutsch et al. (1992) display a morphology different from our near-infrared images. At the longer wavelengths the flux is dominated by thermal dust emission and the images reflect the temperature and density of dust 
in the central regions. It is interesting to note that the data of Deutsch (1990) and Deutsch et al. (1992) indicate that the infrared emission is extended in the north-south direction as expected by the dust distribution found here (Fig. 6). However, their data show that there are density peaks present in the east-west direction and not along the equatorial (N-S) plane.

The greater extent of emission at $K$ relative to $H$ is due to the wavelength dependence of the continuum optical depth, which is lower at the longer wavelength, allowing the scattered photons to escape at an angular distance that is farther from the poles. For a standard interstellar extinction law, our models suggest that $A_{V} \sim 87 \mathrm{mag}$ along the stellar equator. Although uncertain by $\sim 20 \%$ or more, this value is consistent with $A_{V} \sim 70-110 \mathrm{mag}$ along the line of sight as found by Lequeux \& Jourdain de Muizon (1990). We also find the bipolar axis to be inclined to the plane of the sky by $i \sim 45^{\circ}$. This inclination is the same as that found by Carsenty \& Solf (1982) through their analysis of the radial velocity structure. We cannot estimate how much thermal dust emission contributes to the observed flux without polarization measurements. However, since the single-scattering models presented here are a fairly good representation of the emission, the contribution from warm dust is likely to be small. Johnson \& Jones (1991) have recently made polarization measurements of AFGL 618 at $K$ that indicate a large fraction of the emission at $2.2 \mu \mathrm{m}$ is indeed scattered into the line of sight within the visible lobes (see also Dougados \& Rouan 1990).

\section{CONCLUSIONS}

The observations presented in this paper reinforce the picture derived from earlier observations for the structure of AFGL 618. However, the strongly bipolar dust distribution suggested by SC81 (their Fig. 2) and others does not appear to be correct for this object. AFGL 618 seems to be best represented by a dust distribution of the type suggested by Morris (1981) and Yusef-Zadeh et al. (1984) for AFGL 2688. Using this model, we have verified that the total extinction must be large along the stellar equator $\left(\dot{A}_{V} \sim \overline{8} \overline{\mathrm{mag}}\right)$ and low along the poles. Our data are consistent with an inclination to the plane of the sky of $i \sim 45^{\circ}$.

A clumpy medium has been suggested by others (e.g., SC81; Burton \& Geballe 1986) to explain the ionization and velocity structure. Our analysis of the molecular hydrogen fluorescent emission detected in our near-IR spectrum is not inconsistent with this hypothesis. However, there is little new evidence in our data which strongly supports the idea of a clumpy medium. What is required, however, is that the observed molecular region be shielded from direct exposure to the UV flux of the central star. The $\mathbf{H}_{2}$ emission in our spectrum is dominated by emission from collisionally excited molecules at an excitation temperature of $T_{\text {ex }} \sim 2000 \mathrm{~K}$, which is consistent with excitation in shocks (see also Jura \& Kroto 1990). Such emission had previously been detected at $\lambda \sim 2 \mu \mathrm{m}$ (e.g., Thronson 1981; Beckwith et al. 1984; Burton \& Geballe 1986).

A complex distribution of gas and dust is vividly displayed in both spectrum and images. Since this clearly is a rapidly evolving object at one of the most mysterious points in stellar evolution, continued monitoring of key features is important. As material flows away from the central star, its density will drop. Obscuration of UV starlight will decrease. Perhaps fluorescent emission from molecular hydrogen will increase. This type of emission has been observed from at least one other compact young planetary nebula, Hubble 12 (Dinerstein et al. 1988). It is important to note that the wavelength range accessible to the Ge spectrometer is particularly useful for discriminating fluorescent from thermal $\mathrm{H}_{2}$ emission. A survey for this type of emission in the nearest PPNe and young PNe is presently being carried out. The new data should provide additional insight into the evolution of these objects.

The authors would like to thank G. Schmidt, H.-W. Rix, H. A. Thronson, M. Merrifield, P. G. Martin, and L. Deutsch for useful discussions and comments; L. Deutsch also kindly made her thesis data available to us. T. Greene was of great help with reduction of the image data. We also thank the referee for suggestions concerning the interpretation of our infrared images. W. B. L. is pleased to acknowledge support by the National Aeronautics and Space Administration through grant NAGW-763 while at the University of Arizona and the National Sciences and Engineering Research Council of Canada. P. R. M. was supported by the Netherlands Foundation for Pure Research (NWO) under grant number 782-372025, and by the National Academy of Sciences through an NRC Fellowship. J. H. B. thanks NASA for support through grant NAGW-2255 to the University of Arizona. The construction of the spectrometer and the contributions of D. M. K. were supported by the National Science Foundation grant number AST 8715236. We also thank E. F. Montgomery and C. L. Thompson for their work on the instrument.

\section{REFERENCES}

Bachiller, R., Gómez-González, J., Bujarrabal, V., \& Martín-Pintado, J. 1988, A\&A, 196, L5

Balick, B. 1989, in IAU Symp. 131, Planetary Nebulae, ed. S. Torres-Peimbert (Dordrecht: Kluwer), 83

Beckwith, S., Beck, S. C., \& Gatley, I. 1984, ApJ, 280, 648

Black, J. H., \& Dalgarno, A. 1976, ApJ, 203, 132

Black, J. H., \& van Dishoeck, E. F. 1987, ApJ, 322, 412

Bond, H. E., \& Livio, M. 1990, ApJ, 355, 568

Bujarrabal, V., Gómez-González, J., Bachiller, R., \& Martín-Pintado, J. 1988, A\&A, 204, 242

Burton, M. G., \& Geballe, T. R. 1986, MNRAS, 223, 13P

Calvet, N., \& Cohen, M. 1978, MNRAS, 182, 687

Calvet, N., \& Peimbert, M. 1983, Rev. Mexicana Astron. Af., 5, 319

Campins, H., Rieke, G. H., \& Lebofsky, M. J. 1985, AJ, 90, 896

Carsenty, U., \& Solf, J. 1982, A\&A, 106, 307

Cernicharo, J., Guélin, M., Martín-Pintado, J., Penalver, J., \& Mauersberger, R. 1989, A\&A, 222, L1

Deutsch, L. K. 1990, Ph.D. thesis, Harvard Univ.
Deutsch, L. K., Hora, J. L., Hoffman, W. F., \& Fazio, G. G. 1992, in preparation

Dinerstein, H. L., Lester, D. F., Carr, J. S., \& Harvey, P. M. 1988, ApJ, 327, L27

Dougados, C., \& Rouan, D. 1990, in From Miras to Planetary Nebulae: Which Path for Stellar Evolution?, ed. M. O. Mennessier \& A. Omont (Gif sur Yvette: Editions Frontières), 544

Elias, J. H., Frogel, J. A., Mathews, K., \& Neugebauer, G. 1982, AJ, 87, 1029

Field, G. B., Somerville, W. B., \& Dressler, K. 1966, ARA\&A, 4, 207

Gammie, C. F., Knapp, G. R., Young, K., Phillips, T. G., \& Falgarone, E. 1989, ApJ, 345, L87

Glassgold, A. E., Mamon, G. A., \& Huggins, P. J. 1991, ApJ, 373, 254

Gottlieb, E. W., \& Liller, W. 1976, ApJ, 207, L135

Grandi, S. A. 1975, ApJ, 196, 465

Hartquist, T. W., \& Dyson, J. E. 1987, MNRAS, 228, 957

Hoffleit, D., \& Jaschek, C. 1982, Bright Star Catalog (New Haven: Yale Univ. Obs.)

Iben, I., \& Renzini, A. 1983, ARA\&A, 21, 308

Johnson, J. J., \& Jones, T. J. 1991, AJ, 101, 1735 

33

Knapp, G. R., \& Morris, M. 1985, ApJ, 292, 640

Kwok, S., \& Bignell, R. C. 1984, ApJ, 276, 544

Kwok, S., \& Feldman, P. A. 1981, ApJ, 267, L67

Lequeux, J., \& Jourdain de Muizon, M. 1990, A\&A, 240, L19

Livio, M., Salzman, J., \& Shaviv, G. 1979, MNRAS, 188, 1

Lo, K. Y., \& Bechis, K. P. 1976, ApJ, 205, L21

Martin, P. G., \& Rogers, C. 1987, ApJ, 322, 374

Martín-Pintado, J., Bujarrabal, V., Bachiller, R., Gómez-González, J., \& Planesas, P. 1988, A\&A, 197, L15

Morris, M. 1981, ApJ, 249, 572 1987, PASP, 99, 1115

Rieke, G. H., \& Lebofsky, M. J. 1985, ApJ, 288, 618

Rieke, M. J., Rieke, G. H., \& Montgomery, E. F. 1987, in Infrared Astronomy with Arrays, ed. C. G. Wynn-Williams \& E. E. Becklin (Honolulu: Institute for Astronomy, Univ. of Hawaii), 213

Rix, H.-W., Carleton, N. P., Rieke, G., \& Rieke, M. 1990, ApJ, 363, 480

Rowan-Robinson, M., \& Harris, S. 1983, MNRAS, 202, 797

Rudy, R. J., Rossano, G. S., \& Puetter, R. C. 1989, ApJ, 346, 799

Russell, R. W., Soifer, B. T., \& Willner, S. P. 1978, ApJ, 220, 568

Schmidt, G. D., \& Cohen, M. 1981, ApJ, 246, 444 (SC81)

Sternberg, A., \& Dalgarno, A. 1989, ApJ, 338, 197

Thompson, R. 1987, ApJ, 312, 784

Thronson, H. A. 1981, ApJ, 248, 984

Thronson, H. A., Latter, W. B., Black, J. H., Bally, J., \& Hacking, P. 1987, ApJ, 322,770

Thronson, H. A., \& Mozurkewich, D. 1983, ApJ, 271, 611

van Dishoeck, E. F., \& Black, J. H. 1986, ApJS, 62, 109

Volk, K. M., \& Kwok, S. 1989, ApJ, 342, 345

Westbrook, W. E., Becklin, E. E., Merrill, K. M., Neugebauer, G., Schmidt, M. Willner, S. P., \& Wynn-Williams, C. G. 1975, ApJ, 202, 407

Wynn-Williams, C. G. 1977, MNRAS, 181, 61P

Yusef-Zadeh, F., Morris, M., \& White, R. L. 1984, ApJ, 278, 186

Zuckerman, B., \& Gatley, I. 1988, ApJ, 324, 501 